\title{
Reversão de estomia intestinal de eliminação: um olhar para a produção científica
}

\author{
Reversion of intestinal elimination ostomy: a look at scientific production
}

Reversión de la eliminación de la ostomía intestinal: una mirada a la producción científica

Amanda Suélen Monteiro ${ }^{1}$, Evelyn Boeck dos Santos ${ }^{1}$, Bruna Sodré Simon ${ }^{1}$, Cínthia Cristina Oliveski ${ }^{1}$, Angélica Dalmolin ${ }^{1}$, Eduardo da Silva Gomes ${ }^{1}$, Nara Marilene Oliveira Girardon-Perlini ${ }^{1 *}$.

\section{RESUMO}

Objetivo: Caracterizar, através de uma revisão integrativa, por meio de indicadores bibliométricos, a produção científica publicada em periódicos online, acerca da reversão de estomia intestinal de eliminação. Métodos: Estudo documental de revisão de literatura, realizado via Portal Regional da Biblioteca Virtual de Saúde com a estratégia: (tw:("reversão" OR "fechamento")) AND (tw:("colostomia" OR "ileostomia" OR "estomia" OR "ostomia" OR "estoma" OR "ostoma")). Incluíram-se artigos completos, disponíveis online, nos idiomas português, inglês ou espanhol, sem recorte temporal. A análise deu-se pela distribuição de frequência simples e relativa. Resultados: O corpus foi constituído de 41 artigos, dos quais $63,4 \%$ foram localizados na Medical Literature and Retrivial System Online. O ano de 2014 teve o maior número de publicações, com 14,6\% dos artigos, $34,1 \%$ do total dos estudos foram realizados no Brasil e a Revista Brasileira de Coloproctologia obteve o maior número de publicações, com $17 \%$. Todos os estudos foram realizados por médicos. Verificou-se que $80,5 \%$ correspondiam a artigos originais e $48,8 \%$ eram quantitativos. Considerações finais: Os estudos foram desenvolvidos por médicos, direcionados a cirurgia, especialmente, a questões relacionadas às técnicas cirúrgicas e ao pré e pós-operatório. Entende-se que há necessidade de produções que vislumbrem perspectivas do cuidado de enfermagem relativas à temática.

Palavras-chave: Reversão, Estomia, Colostomia, Ileostomia, Enfermagem.

\begin{abstract}
Objective: To characterize, through an integrative review and bibliometric indicators, the scientific production published in online journals about the reversion of elimination intestinal stomies. Method: Documentary literature review study, carried out the Portal Regional da Biblioteca Virtual de Saúdewith the strategy: (tw: ("reversion" OR "closure")) AND (tw:("colostomy" OR "ileostomy" OR "ostomy" OR "stoma" OR "ostoma")). Complete articles have been included, available online, in the Portuguese, English or Spanish languages, without temporal cut. The analysis was based on the distribution of simple and relative frequencies. Results: The corpus consisted of 41 articles, of which $63.4 \%$ were located in the Medical Literature and Retrivial System Online. The year 2014 had the largest number of publications, with $14.6 \%$ of the articles, $34.1 \%$ of the studies were conducted in Brazil and the RevistaBrasileira de Coloproctologia obtained the highest number of publications, with $17 \%$. All studies were performed by physicians. It was verified that $80.5 \%$ corresponded to original articles and $48.8 \%$ were quantitative. Conclusion: The studies were developed by physicians, directed to surgery, especially, to questions related to surgical techniques and to the pre and postoperative. It is understood that there is a need for productions that glimpse nursing care perspectives related to this theme.
\end{abstract}

Key words: Reversal, Ostomy, Colostomy, lleostomy, Nursing.

1Universidade Federal de Santa Maria (UFSM), Santa Maria - RS. *E-mail: nara.girardon@gmail.com SUBMETIDO EM: 4/2020 


\section{RESUMEN}

Objetivo: Caracterizar, a través de una revisión integradora e indicadores bibliométricos, la producción científica publicada en revistas en línea, sobre la reversión de la eliminación de la ostomía intestinal. Métodos: Estudio de revisión de literatura documental, realizado a través del Portal Regional de la Biblioteca Virtual en Salud con la estrategia: (tw :( "reversión" O "cierre")) Y (tw :( "colostomía" O "ileostomía" O "estoma" O "ostomía" O "estoma" O "ostoma")). Los artículos completos estaban disponibles, disponibles en línea, en portugués, inglés o español, sin marco de tiempo. El análisis se realizó mediante la distribución de frecuencia simple y relativa. Resultados: El corpus consistió en 41 artículos, $63.4 \%$ de los cuales se ubicaron en el Sistema de literatura médica y recuperación en línea. El año 2014 tuvo el mayor número de publicaciones, con el $14,6 \%$ de los artículos, el $34,1 \%$ de los estudios se realizaron en Brasil y la Revista Brasileira de Coloproctologia obtuvo el mayor número de publicaciones, con el $17 \%$. Todos los estudios fueron realizados por médicos. Se encontró que el $80.5 \%$ correspondía a artículos originales y el $48.8 \%$ eran cuantitativos. Consideraciones finales: Los estudios fueron desarrollados por médicos, dirigidos a la cirugía, especialmente a cuestiones relacionadas con las técnicas quirúrgicas y pre y postoperatorias. Se entiende que existe la necesidad de producciones que prevean perspectivas de la atención de enfermería relacionadas con este tema.

Palabras clave: Reversión, Estomía, Colostomía, Ileostomia, Enfermería.

\section{INTRODUÇÃO}

Estomia é uma palavra de origem grega que significa boca ou abertura em qualquer víscera oca do organismo, a qual é confeccionada cirurgicamente e permite a comunicação do meio interno do corpo com o meio externo (SANTOS OJ, et al., 2016). As estomias mais recorrentes são as intestinais de eliminação, com destaque para as colostomias e as ileostomias, realizadas no intestino grosso e intestino delgado, respectivamente (LUZ ALA, et al., 2014).

A confecção de um estoma pode ser requerida por diversas causas, dentre as principais estão as anomalias congênitas, os traumas abdominais, as doenças inflamatórias intestinais e, ainda, as neoplasias, sendo a mais predominante o câncer colorretal. A partir da patologia e das condições clínicas do indivíduo elas podem ser classificadas como temporária ou definitiva (SANTOS VLCG e CESARETTI IUR, 2015). Os estomas definitivos ocorrem quando não existe a possibilidade de restabelecer o trânsito intestinal, enquanto os temporários objetivam proteger uma anastomose (OLIVEIRA G, et al., 2010). O tempo para a realização da reversão ainda é muito discutido, pois alguns cirurgiões acreditam que o fechamento precoce do estoma é a melhor opção, enquanto que para outros, o tempo ideal é após a resolução total do cenário inflamatório no abdômen e a melhora na nutrição do paciente (FONSECA AZ, et al., 2017).

Convém destacar que existem diferentes tipos de estoma intestinal de eliminação, tais como: estomia terminal, estomia em alça e estomia em duas bocas (dupla) (SANTOS VLCG e CESARETTI IUR, 2015). As estomias terminais comumente são definitivas, com exceção dos casos da técnica de Hartmann, que permitem a reversão. As estomias em alça geralmente são temporárias, possibilitando a sua reversão por meio da enterorrafia (no íleo) e da colorrafia (no cólon), quando realizadas no intestino grosso, normalmente estão localizadas no cólon transverso. As estomias em duas bocas são constituídas por dois estomas completamente separados, os quais são exteriorizados justapostos no mesmo orifício na forma de cano de escopeta ou exteriorizados no abdômen em locais distantes e o segmento distal é denominado de fístula mucosa (SANTOS VLCG e CESARETTI IUR, 2015; FONSECA AZ, et al., 2017; SMELTER SC e BARE BG, 2015).

É consenso entre os profissionais de saúde, e a literatura confirma, que a pessoa com estoma sofre modificações fisiológicas, psicossociais e emocionais, uma vez que precisa apreender a conviver com uma nova condição de vida. Assim, faz-se necessário incorporar novas práticas de autocuidado e diferentes hábitos, a fim de amenizar as alterações corporais decorrentes desta situação (SIMON BS, et al., 2015). Nessa esfera, a gestão do cuidado abrange múltiplas dimensões, tais como: a individual, a familiar, a 
profissional, a organizacional, a sistêmica e a societária, o que permite, a partir das demandas apresentadas pelas pessoas com estoma, atuar de forma a incentivar sua autonomia, proporcionando bem-estar e uma vida produtiva (TRAMONTINA PC, et al., 2019). A confluência destas dimensões possibilita facilitar a reabilitação e refletir beneficamente na qualidade de vida.

Para deixar de conviver com o estoma, quando possível, há necessidade de estas pessoas sejam submetidas à cirurgia de reversão e reconstrução intestinal. Para isso, destaca-se que a identificação antecipada das complicações é essencial para diminuir os riscos que permeiam esse procedimento cirúrgico e obter melhores resultados, tendo em vista que o objetivo da reversão é proporcionar melhora na qualidade de vida da pessoa (FONSECA AZ, et al., 2017).

Nesse contexto, justifica-se a relevância deste estudo a partir da necessidade de conhecer, quantitativamente, as características das produções científicas acerca da reversão de estoma intestinal de eliminação. Para tanto, busca-se responder à questão de pesquisa: "Quais as características das publicações científicas relacionadas à reversão de estomia intestinal de eliminação?". O objetivo deste estudo foi caracterizar, por meio de indicadores bibliométricos, a produção científica publicada em periódicos online, acerca da reversão de estomia intestinal de eliminação.

\section{MÉTODOS}

Trata-se de um estudo documental de revisão integrativa de literatura, realizado via Portal Regional da Biblioteca Virtual de Saúde (BVS). A bibliometria foi escolhida, pois possibilita mensurar os diferentes aspetos relativos às produções científicas de um determinado tema, agrupando sinteticamente os resultados. Entre os objetivos da bibliometria, destaca-se o de identificar as tendências e o crescimento do conhecimento em uma área específica (MEDEIROS JMG e VITORIANO MAV, 2015).

Para a elaboração do estudo organizou-se três etapas, sendo a primeira o levantamento bibliográfico, realizado em março de 2020, utilizando a estratégia de busca: (tw: ("reversão" OR "fechamento")) AND (tw: ("colostomia" OR "ileostomia" OR "estomia" OR "ostomia" OR "estoma" OR "ostoma")). A escolha destes descritores e/ou palavras-chaves visou localizar o maior número possível de artigos sobre a temática, não sendo estabelecido um recorte temporal, a fim de minimizar possíveis perdas.

Para selecionar a amostra, elencaram-se como critérios de inclusão: estudos com resumo completo, artigo disponível online na íntegra, publicados nos idiomas português, inglês ou espanhol referentes à temática de reversão de estomia intestinal de eliminação. Foram excluídas as dissertações, teses e manuais. As publicações repetidas foram contabilizadas apenas uma vez. A seleção dos estudos foi desenvolvida de modo duplo independente, com vistas a evitar possíveis vieses nesta etapa.

A busca resultou em 145 estudos, sendo que, após a leitura dos títulos e resumos, 66 foram excluídos: 33 por apresentarem resumo incompleto; 28 por não atenderem à temática do estudo; três por não estarem nos idiomas elencados; e dois artigos foram contabilizados uma vez devido duplicação nas bases de dados. Assim, a primeira etapa resultou em 79 artigos pré-selecionados.

Posteriormente, realizou-se a leitura na íntegra, culminando na exclusão de 38 estudos em que 28 não correspondiam à temática e dez não apresentavam texto completo online. Houve divergência referente à inclusão ou exclusão de 24 artigos pelo primeiro revisor. Para tanto, os mesmos foram lidos na íntegra por um segundo revisor, com domínio no assunto, visando reduzir o risco de perdas de publicações relevantes. Assim, os dois revisores chegaram ao consenso de que estes 24 artigos não abordavam a temática deste estudo. O corpus final correspondeu a 41 artigos relacionados à reversão de estomias intestinais de eliminação (Figura 1).

A segunda etapa do estudo constituiu-se na organização e análise dos dados, sendo que as informações foram extraídas e organizadas em uma planilha, elaborada exclusivamente para o estudo, composta pelas variáveis: base de dados/bibliotecas digitais; ano; procedência geográfica; idioma; periódico de publicação; categoria profissional dos autores; modalidade de estudo (original/revisão); característica metodológica; população do estudo; tema central; e descritores. 
Por fim, procedeu-se a terceira e última etapa, constituída na apresentação e análise dos resultados. Para tanto, calculou-se a distribuição das frequências em números absolutos e relativos. A partir da leitura dos artigos buscou-se identificar o assunto principal abordado em cada um e, então, organizou-se tópicos por similaridade de conteúdo. Assim, considerando os temas originados construiu-se um diagrama referente aos assuntos centrais dos artigos.

Figura 1: Fluxograma do processo de seleção dos estudos incluídos na revisão de literatura.

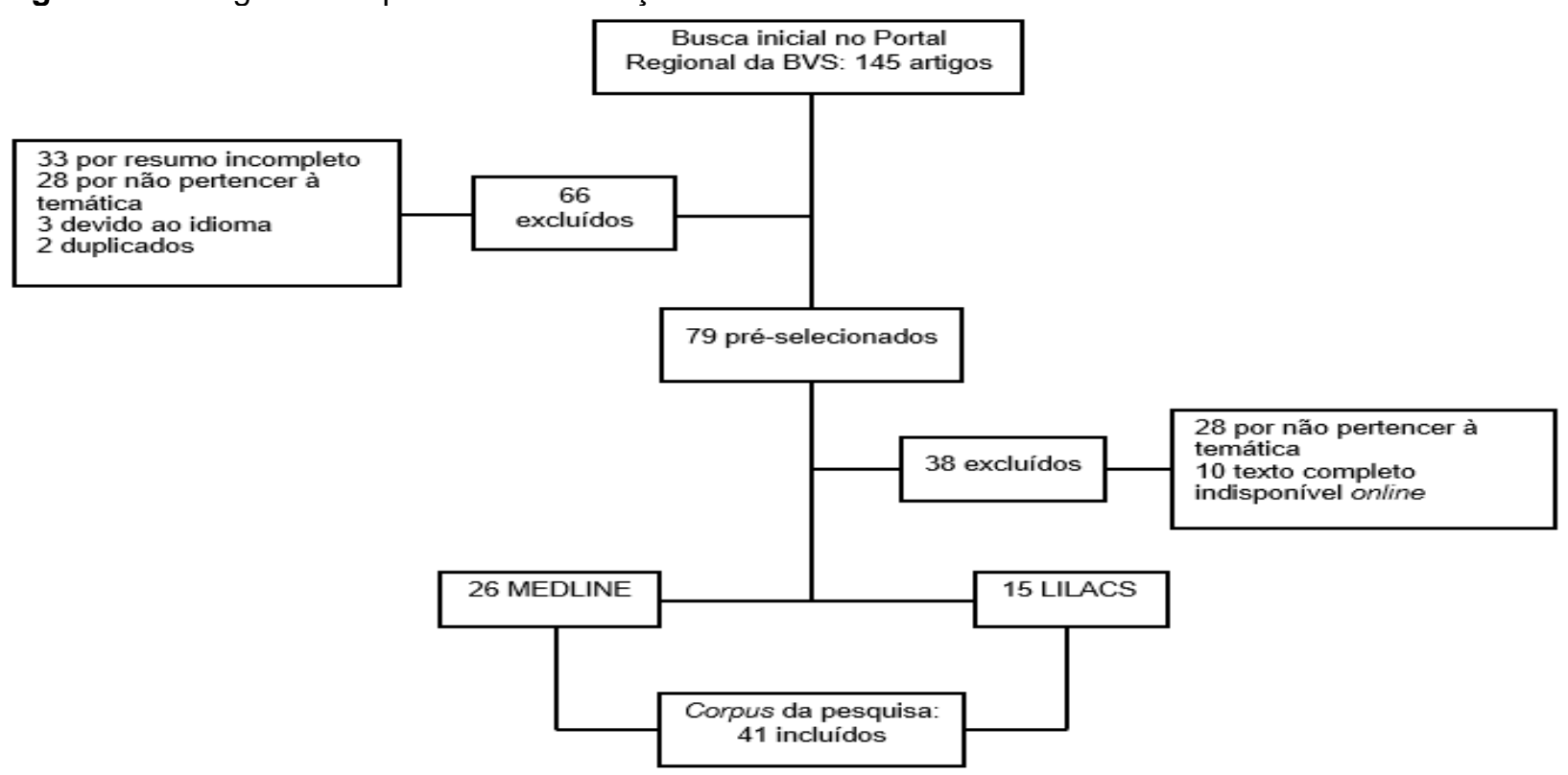

Fonte: Monteiro AS, et al., 2020.

\section{RESULTADOS}

O corpus do estudo foi composto por 41 artigos. No que diz respeito à base de dados das publicações, houve predomínio de 26 (63,4\%) artigos na Medical Literature and Retrivial System Online (MEDLINE), seguido de 15 (36,6\%) na Literatura Latino-Americana e do Caribe em Ciências da Saúde (LILACS).

Constatou-se que o ano de 2014 obteve o maior número de publicações abordando a temática relacionada a reversão de estomia intestinal de eliminação, com seis (14,6\%) estudos, seguido por 2013, 2015 e 2017 com cinco (12,2\%) publicações cada (Figura 2).

Figura 2: Artigos selecionados com relação ao ano de publicação.

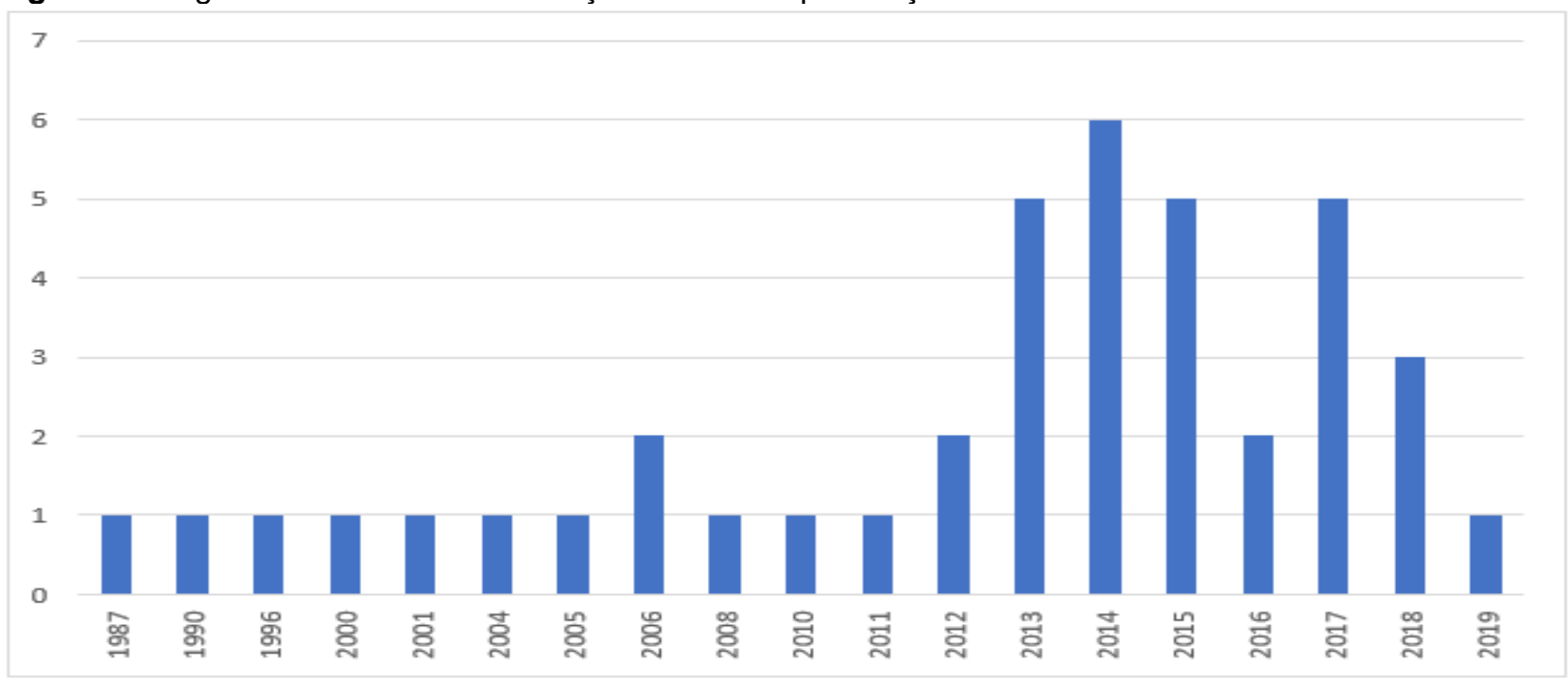

Fonte: Monteiro AS, et al., 2020. 
As pesquisas publicadas tiveram como predominância de origem o Brasil, com 14 (34,3\%) artigos, seguido pelos Estados Unidos e Alemanha, com quatro estudos (9,7\%); Espanha, Inglaterra e Itália cada um com três publicações (7,3\%); Austrália e Holanda com dois estudos cada (4,8\%); Coréia, Filipinas, Suíça e Irlanda com um $(2,5 \%)$ artigo cada e dois $(4,8 \%)$ estudos não constavam a procedência geográfica.

Observa-se que dentre os 14 (34,3\%) estudos realizados no Brasil, houve predomínio de publicações oriundas da região sudeste, especialmente dos estados de São Paulo com sete (50\%) artigos, Rio de Janeiro e Minas Gerais com dois artigos cada (14,3\%). Os demais foram procedentes da região sul do país, sendo dois $(14,3 \%)$ do Paraná e um $(7,2 \%)$ do Rio Grande do Sul.

Com relação ao idioma de publicação, 27 (65,8\%) eram em inglês, 11 (26,8\%) em português, um (2,5\%) em espanhol e dois (4,9\%) nos idiomas inglês e português. O periódico que obteve maior número de publicações acerca da temática foi a Revista Brasileira de Coloproctologia com sete (17\%) artigos, seguido do periódico Colorectal Disease com quatro $(9,7 \%)$ estudos, de acordo com o apresentado na (Tabela 1).

Tabela 1: Distribuição dos artigos em relação ao periódico.

\begin{tabular}{|c|c|c|}
\hline Periódicos & $\mathbf{N}$ & $\%$ \\
\hline Revista Brasileira de Coloproctologia & 7 & 17,0 \\
\hline Colorectal Disease & 4 & 9.7 \\
\hline International Journal of Surgery & 3 & 7,2 \\
\hline Journal of Coloproctology & 3 & 7,2 \\
\hline Revista do Colégio Brasileiro de Cirurgiões & 3 & 7,2 \\
\hline Arquivos Brasileiros de Cirurgia Digestiva & 2 & 4,8 \\
\hline Diseases of the colon \& Rectum & 2 & 4,8 \\
\hline International Journal of Colorectal Disease & 2 & 4,8 \\
\hline Techniques in Coloproctology & 2 & 4,8 \\
\hline Acta Cirúrgica Brasileira & 1 & 2,5 \\
\hline Archives of Disease in Childhood & 1 & 2,5 \\
\hline British Journal of Surgery & 1 & 2,5 \\
\hline Cirugía Española & 1 & 2,5 \\
\hline Digestive Surgery & 1 & 2,5 \\
\hline Fortschritte auf dem Gebiete der Röntgenstrahlen & 1 & 2,5 \\
\hline Langenbeck's Archives of Surgery & 1 & 2,5 \\
\hline Ostomy Wound Manage & 1 & 2,5 \\
\hline Pediatric Surgery International & 1 & 2,5 \\
\hline Revista Brasileira de Anestesiologia & 1 & 2,5 \\
\hline Revista Española de Enfermedades Digestivas & 1 & 2,5 \\
\hline Surgical Endoscopy & 1 & 2,5 \\
\hline World Surgery Journal & 1 & 2,5 \\
\hline Total & 41 & 100,0 \\
\hline
\end{tabular}

Fonte: Monteiro AS, et al., 2020.

Constatou-se que todos os estudos foram realizados por médicos. No que concerne à modalidade do estudo, verificou-se a predominância de $33(80,5 \%)$ artigos originais, sete $(17,07 \%)$ artigos de revisão e um $(2,43 \%)$ relato de caso. Em relação à característica metodológica, $20(48,78 \%)$ eram estudos quantitativos, $14(34,15 \%)$ qualitativos e sete $(17,07 \%)$ estudos de revisão. 
No que se refere à fonte das informações utilizadas nas publicações, identificou-se que $21(51,22 \%)$ foram desenvolvidas com pacientes, 11 (26,83\%) utilizaram prontuários, sete $(17,07 \%)$ analisaram artigos, um $(2,44 \%)$ investigou prontuários e pacientes, e um $(2,44 \%)$ dos estudos analisou imagens fotográficas. Verificou-se que os artigos versavam sobre diferentes temas, no entanto, a ênfase centrou-se no procedimento cirúrgico de reversão da estomia (Figura 3).

Figura 3: Temas centrais dos estudos referentes à reversão de estomia intestinal de eliminação.

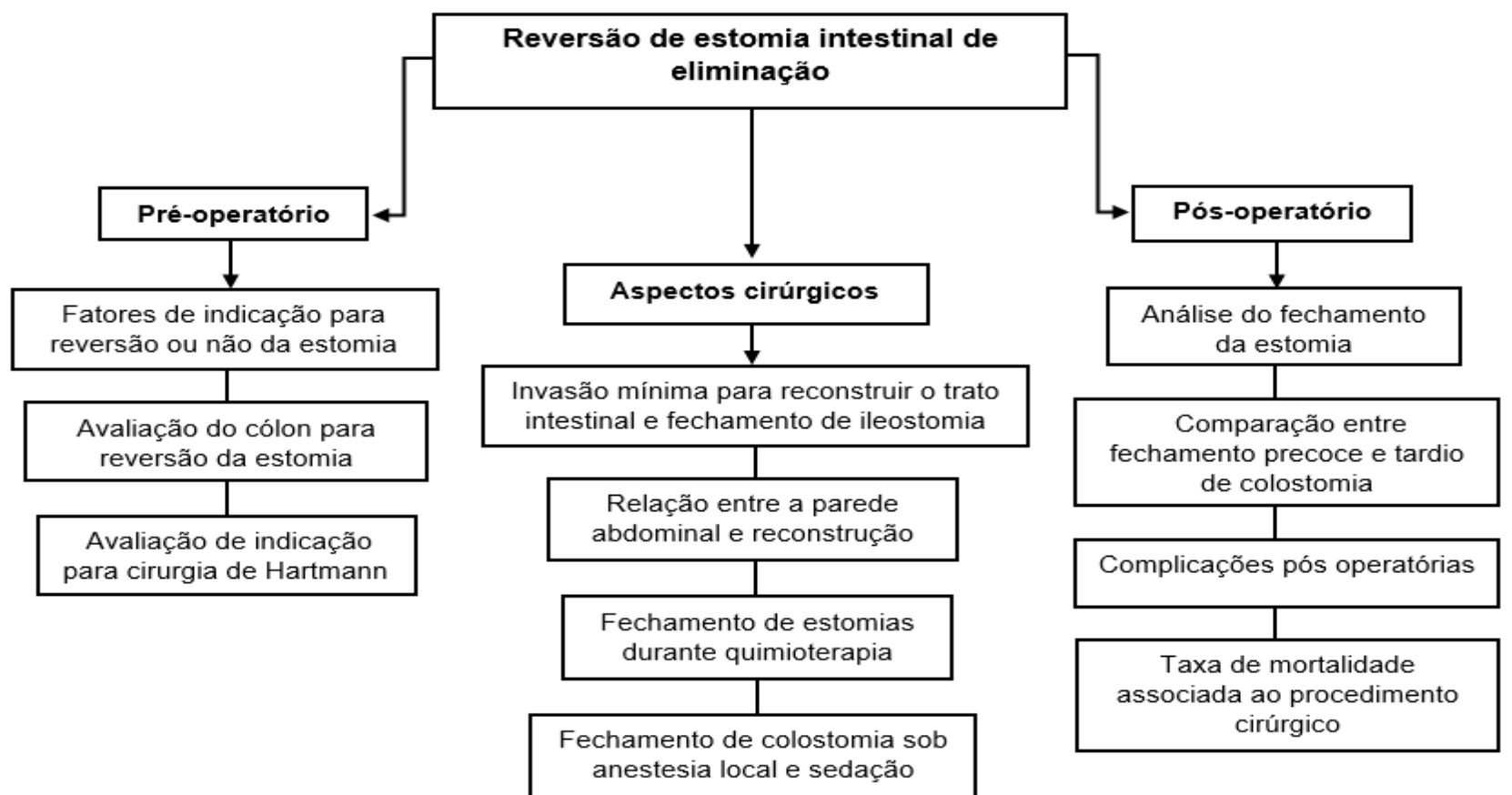

Fonte: Monteiro AS, et al., 2020.

\section{DISCUSSÃO}

A predominância da base de dados MEDLINE, com maior número de estudos em comparação a LILACS, está relacionada ao fato de ser considerada uma das bases mais antigas, publicando estudos desde 1948, procedentes de diversos países, com predomínio da língua inglesa (NATIONAL LIBRARY OF MEDICINE).

Com base nos resultados obtidos, o primeiro estudo que menciona a reversão de estomia intestinal foi publicado em 1987, o qual trata de experiências de serviços em relação a pacientes com quadro clínico de obstrução intestinal completa e as opções cirúrgicas aliadas às possíveis complicações. Para isso, analisouse retrospectivamente os prontuários de 63 pacientes portadores de câncer de cólon e reto que foram divididos em grupos, de acordo com o procedimento a que seriam submetidos: ressecção de anastomose primária; colostomia, ressecção e fechamento de colostomia; ressecção com colostomia e fechamento da colostomia posterior; e colostomia (WIEDERKEHR JC, et al., 1987). No entanto, é perceptível a escassez de publicações de estudos com relação a essa temática nos anos subsequentes, sendo possível verificar um crescimento a partir de 2011.

A confecção das primeiras estomias intestinais de eliminação datam a partir de 1700, sendo idealizada e modificada por diferentes médicos que realizaram este procedimento cirúrgico, embora seja controverso, na literatura, quando se realizou a primeira estomia. Ao longo dos anos constata-se a proposição de diversas técnicas cirúrgicas, buscando a melhor maneira de exteriorizar alças intestinais e facilitar o manuseio após a alta hospitalar. Em consonância percebe-se o desenvolvimento de equipamentos coletores e o aumento das publicações relacionadas às necessidades psicossociais da pessoa com estomia (SANTOS VLCG e CESARETTI IUR, 2015). No entanto, não se localizou registros históricos a respeito de quando se iniciam as cirurgias de reversão de estomias, mas acredita-se que com o avanço dos produtos médicos, a exemplo dos grampeadores circulares nos anos de 1970, ocorreu o aprimoramento das técnicas cirúrgicas, possibilitando a anastomose do sistema gastrointestinal com maior facilidade (FONSECA LM, et al., 2018). 
Embora os estudos tenham sido realizados em diferentes países, os resultados apontam o Brasil como o país com maior número de publicações. Além disso, notou-se um aumento no quantitativo de estudos publicados, sobretudo, na última década, fato que pode estar relacionado com a implementação, pelo Ministério da Saúde, da Resolução oㅡ 400 de 2009, a qual trata das diretrizes nacionais para uma assistência de qualidade, maior visibilidade às pessoas com estomias e propõe indicações relacionadas ao estabelecimento de fluxos de atendimento para as cirurgias de reversão (BRASIL, 2009).

Nesse contexto, essa legislação, em especial, pode ter contribuído para o aumento das pesquisas na área da coloproctologia, direcionando estudos para o fechamento das estomias e reconstrução do trânsito intestinal. A nível nacional, constatou-se a concentração de trabalhos publicados na região sudeste. Esse fato pode ser explicado a partir do alto índice de desenvolvimento e, consequentemente, maior quantidade de instituições de ensino superior nesta região, as quais têm em sua missão institucional o desenvolvimento do ensino, da pesquisa e da extensão que associados ao incentivo de instituições privadas fomenta a produção do conhecimento científico (SIDONE OJG, et al., 2016).

Ainda que a maioria dos estudos seja de origem brasileira, evidenciou-se o idioma inglês como o mais prevalente. Este fato está diretamente relacionado com a publicação das pesquisas em periódicos internacionais e, também, por ser considerado o idioma que possibilita maior alcance e divulgação dos estudos, bem como a disseminação de novos conhecimentos e contribuições no que tange a internacionalização para as pesquisas científicas. A Revista Brasileira de Coloproctologia e o Colorectal Disease destacam-se como periódicos que publicam estudos científicos relacionados à reversão de estomia intestinal. Os meios de divulgação estão voltados para a área da especialidade, publicando trabalhos científicos relacionados à medicina e cirurgia humanas voltadas para a coloproctologia e gastroenterologia, elaborados por especialistas nacionais e estrangeiros.

Considerando os avanços tecnológicos e o desenvolvimento de abordagens voltadas às técnicas para reversão da estomia intestinal de eliminação, evidenciou-se que a elaboração dos artigos analisados neste estudo, em sua totalidade são conduzidos por médicos, o que demonstra a consonância entre os estudos realizados, o interesse em técnicas cirúrgicas e os aspectos clínicos relativos a esse procedimento. Ao analisar-se o conjunto dos periódicos em que as pesquisas foram publicadas constata-se que $50 \%$ estão relacionados a área da cirurgia, o que se mostra em acordo com a formação dos autores dos artigos e a abordagem dos estudos. Dessa forma, pode-se evidenciar a presença de lacuna na construção do conhecimento nessa temática, uma vez que não se localizou produções de autoria de outros profissionais de saúde e nem publicações dissociadas de periódicos da medicina.

Contudo, a Portaria ํㅜ 400 de 2009 estabelece como premissa que as pessoas com estomia precisam ser assistidas por uma equipe multidisciplinar, que objetive a sua reabilitação, a partir da educação em saúde voltada para o autocuidado, a prevenção de complicações e o fornecimento de equipamentos adequados (BRASIL, 2009). Neste sentido, ressaltam-se os serviços especializados de atenção à pessoa com estomia compostos por uma equipe multiprofissional, que devem promover capacitações para os demais profissionais de saúde sobre a adequada conduta pré e pós-operatórias para confecção de estomas, como também para a realização da reconstrução do trânsito intestinal e possíveis tratamentos em casos de complicações pósoperatórias (BRASIL, 2009).

Além da assistência multidisciplinar, a pessoa após a reversão da estomia também necessita da resposta dos diferentes serviços de saúde, com vistas a solucionar os problemas que podem se apresentar no transcorrer do processo de reabilitação, os quais podem ser cíclicos e/ou duradouros. Assim, observa-se que ações realizadas de forma isolada podem não proporcionar a resolutividade total das necessidades dessas pessoas, fazendo com que seja preciso a articulação dos diferentes níveis do sistema de saúde, a fim de tecer uma Rede de Atenção à Saúde (RAS) (TRAMONTINA PC, et al., 2019).

Um dos objetivos da RAS refere-se à atenção integral à saúde, o que possibilita a reflexão sobre a necessidade de organizar a gestão do cuidado em saúde voltado para as questões de prevenção, promoção e reabilitação. Para isso, ao tratar de pessoas que irão vivenciar a reversão da estomia de eliminação intestinal, deve-se atentar para a visualização da pessoa como um todo, envolvendo a integralidade das ações e serviços de saúde, reconhecendo suas reais necessidades, além de primar pela continuidade desse cuidado 
e auxiliar no processo de aceitação da nova realidade (MENDES EV, 2011; POLETTO DB, et al., 2013; TRAMONTINA PC, et al., 2019).

Com isso, percebe-se a necessidade do olhar interdisciplinar para situações que exigem a atuação colaborativa entre diferentes profissionais, em especial enfermeiros estomaterapeutas, uma vez que, o cuidado após a reversão exige conhecimento técnico-científico e, a estomaterapia é uma especialidade privativa da enfermagem, que presta cuidados contínuos nos diferentes níveis de atenção em saúde, tanto à pessoa com estomia, quanto a sua família. Este fato ressalta a importância da investigação e publicação de resultados de pesquisa científicas que embasem o planejamento da assistência, considerando os diversos fatores que podem influenciar no procedimento de reconstrução do trânsito intestinal e a possibilidade de uma reabilitação mais efetiva (AGUIAR JC, et al., 2018).

A prevalência da publicação de estudos originais evidencia a tendência em divulgar resultados inéditos das pesquisas científicas, seja pelos resultados obtidos ou pelo método/técnica utilizado. Assim, entende-se por estudo original, aqueles que apresentam características inovadoras que possuem o objetivo de aumentar e solidificar a construção do conhecimento, a partir de interações entre os aspectos que levaram aos acontecimentos ou à descoberta de novas realidades, a fim de enriquecer a área da pesquisa acerca de tal temática (RODRIGUES GGF, et al., 2017). Com relação à prevalência do método quantitativo adotado na condução dos estudos selecionados, vale ressaltar que esse tem como característica uma abordagem estruturada, que visa gerar dados quantificáveis, com resultados precisos e confiáveis (FERNÁNDEZ SP e DÍAZ SP, 2002). Esse tipo de metodologia se coaduna com os objetivos das publicações analisadas, já que estas, em sua maioria, estavam relacionadas à estudos clínicos e epidemiológicos, cuja coleta de dados teve ênfase em pacientes.

No tocante à distribuição dos estudos com relação aos temas centrais abordados, observa-se pela elaboração do diagrama (Figura 3) a tendência das temáticas estudadas, estando relacionada ao período pré e pós-operatório e aspectos cirúrgicos, direcionando-se a particularidades intrínsecas e extrínsecas que envolvem esse procedimento. Considerando o exposto, percebe-se o predomínio de estudos direcionados aos aspectos cirúrgicos e suas complicações, abordando os fatores pré e pós-operatório, bem como as técnicas implementadas durante a cirurgia de reversão. Assim, não se identificou publicações que explorem as perspectivas de outros profissionais de saúde, que participam do processo de cuidar de pessoas que são submetidas a reversão de estomia intestinal de eliminação, em especial da enfermagem, bem como das vivências e experiências dos pacientes e seus familiares.

Como limitação do estudo destaca-se possíveis vieses advindos da escolha das estratégias de busca e das bases de dados acessadas, que podem ter restringido a captura da totalidade dos trabalhos. Contudo, os resultados encontrados apontam uma lacuna no conhecimento, evidenciando a necessidade de incentivar a produção científica relativa a esta temática, com vistas a ampliar e aprofundar conhecimento.

\section{CONSIDERAÇÕES FINAIS}

Conclui-se que a temática de reversão de estomia intestinal de eliminação está sendo discutida prioritariamente à luz da literatura médico cirúrgica. Apesar de observar a necessidade de produções advindas das outras categorias profissionais, enfatiza-se a relevância de estudos desenvolvidos por enfermeiros generalistas e também, estomaterapeutas, uma vez que, o cuidado durante a reversão exige conhecimento técnico-científico especializado. A escassez de publicações no que concerne às práticas de cuidado direcionadas à pessoa pós-reversão de estomia intestinal e a sua família acarreta um déficit de atualizações sobre o assunto, refletindo um conhecimento ainda incipiente por parte dos profissionais de saúde que prestam essa assistência. Além disso, sabe-se que o pós-operatório de reversão da estomia, impõe condições novas ao cotidiano, necessitando também ser pesquisado, para além das dimensões clínicas e epidemiológicas.

\section{AGRADECIMENTOS E FINANCIAMENTO}

Agradecemos aos Programas PROBIC-FAPERGS, PIBIC-CNPq e FIPE SÊNIOR-UFSM pela concessão de bolsas de Iniciação Científica. 


\section{REFERÊNCIAS}

1. AGUIAR JC, et al. Reconstrução de trânsito intestinal: fatores que influenciam a realização. Revista Eletrônica de Enfermagem, 2018; 20(20): a32.

2. BRASIL. Ministério da Saúde. 2009. Portaria no 400 da Secretaria de Atenção à Saúde, de 16 de novembro de 2009. Estabelece diretrizes nacionais para a atenção à saúde das pessoas ostomizadas no âmbito do Sistema Único de Saúde-SUS, a serem observadas em todas as unidades federadas, respeitadas as competências das três esferas de gestão. Brasília: Diário Oficial da União da República Federativa do Brasil. Disponível em:http://bvsms.saude.gov.br/bvs/saudelegis/sas/2009/prt0400_16_11_2009.html. Acesso em: 28 mar. 2020.

3. FERNÁNDEZ SP, DÍAZ SP. Investigación cuantitativa y cualitativa. Caderno da Atenção Primária, 2002; 9: 76-8.

4. FONSECA AZ, et al. Fechamento de colostomia: fatores de risco para complicação. Arquivos Brasileiros de Cirurgia Digestiva, 2017; 30(4): 231-4.

5. FONSECA LM, et al. Fatores associados à não reconstrução do trânsito intestinal em pacientes com câncer retal submetidos à ressecção anterior do reto e ileostomia de proteção. Revista do Colégio Brasileiro de Cirurgiões, 2018; 45(6): e1998.

6. LUZ ALA, et al. Perfil de pacientes estomizados: revisão integrativa da literatura. Cultura de los Cuidados, 2014; 18(39): 115-23.

7. MEDEIROS JMG, VITORIANO MAV. A evolução da bibliometria e sua interdisciplinaridade na produção científica brasileira. Revista Digital de Biblioteconomia \& Ciência da Informação, 2015; 13(3): 491-503.

8. MENDES EV. As redes de atenção à saúde. Brasília: Organização Pan-Americana da Saúde, 2011; 549p. Disponível em: http://bvsms.saude.gov.br/bvs/publicacoes/redes_de_atencao_saude.pdf Acesso em: 25 mai. 2020.

9. NATIONAL LIBRARY OF MEDICINE. Ficha Informativa Medline. Disponível em: https://www.nlm.nih.gov/pubs/factsheets/medline.html.Acesso em: 28 mar. 2020.

10. OLIVEIRA G, et al. Impacto da estomia: sentimentos e habilidades desenvolvidos frente à nova condição de vida. Revista Estima, 2010; 8(1).

11.POLETTO DB, et al. Vivendo com estoma intestinal: a construção da autonomia para o cuidado. Revista LatinoAmericana de Enfermagem, 2013; 21 (2): 531-538.

12.RODRIGUES GGF, et al. Palliative care for oncological patient: bibliometric study. Revista de Enfermagem UFPE online, 2017; 11(Supl 3): 1349-56.

13.SANTOS OJ, et al. Children and adolescents ostomized in a reference hospital. JournalofColoproctology, 2016; 36(2): 75-9.

14.SANTOS VLCG, CESARETTI IUR. Epidemiologia das estomias. Assistência em estomaterapia: cuidando de pessoas com estomia. São Paulo: Atheneu, 2015; 15p.

15.SANTOS VLCG, CESARETTI IUR. Evolução da enfermagem em Estomaterapia no decorrer de sua história. Assistência em estomaterapia: cuidando de pessoas com estomia. São Paulo: Atheneu, 2015; $1 \mathrm{p}$.

16.SIDONE OJG, et al. A ciência nas regiões brasileiras: evolução da produção e das redes de colaboração científica. Transinformação, 2016; 28(1): 15-32.

17. SIMON BS, et al. "Sempre ajudando em uma coisa ou outra": rede social da família da pessoa com estomia. Revista Eletrônica de Enfermagem, 2015; 17(2): 370-8.

18.SMELTER SC, BARE BG. Tratado de enfermagem médico-cirúrgica. 13 ed. Rio de Janeiro: Guanabara Koogan, 2015.

19.TRAMONTINA PC, et al. Gestão do cuidado à pessoa com estomia e a rede de atenção à saúde. Revista Cuidarte, 2019; 10(1): e613.

20. WIEDERKEHR JC, et al. Tratamento cirúrgico da oclusão intestinal por câncer de cólon e reto. Revista Brasileira de Coloproctologia, 1987; 7(4): 135-8. 